$10.1117 / 2.1200905 .1594$

\title{
Maskless multibeam laser irradiation enables large-area nanostructure fabrication
}

Minghui Hong, Leng Seow Tan, Boris Luk'yanchuk, Luping Shi, and Tow Chong Chong

Laser-interference and laser microlens-array lithography provide significant resolution advances towards high-speed nanomanufacturing.

Compared to other precision-engineering tools, laser irradiation has the unique advantages — as a noncontact process — of being flexible to set up and having high-speed processing capabilities in air, vacuum, or liquid environments. Therefore, laser precision engineering plays an important role in a range of industries. Importantly, lasers can be easily focused to perform smallscale procedures such as marking, drilling, recrystalizing amorphous silicon, and surface cleaning and modification, making them uniquely suited to nanoscale fabrication processes. However, to cater to the demands for ever-smaller processing resolutions, the limits imposed by optical diffraction must be overcome.

We recently demonstrated that lasers operating in the near field can achieve feature resolutions equivalent to that of electron-beam lithography. ${ }^{1}$ For example, 532nm/7ns neodymium-doped yttrium aluminium garnet laser irradiation using an atomic-force-microscopy tip, 400nm/100fs lasing with a near-field scanning-optical-microscopy tip, and excimer-laser irradiation through $1 \mu \mathrm{m}$ transparent particles self-assembled on a substrate surface can achieve resolutions as low as 10, 20, and lower than 50nm, respectively. However, these approaches are limited by critical technical challenges including extremely slow optical scanning speeds. This makes it difficult to apply them to large-area, low-cost nanomanufacturing.

We have been exploring laser-interference lithography (LIL) for large-area, maskless, and noncontact nanofabrication. The underlying principle is based on interference of two coherent light beams that form a horizontal standing-wave pattern. This interference pattern is used to record the patterns on the exposed photoresist. For two-beam interference, the standing wave forms

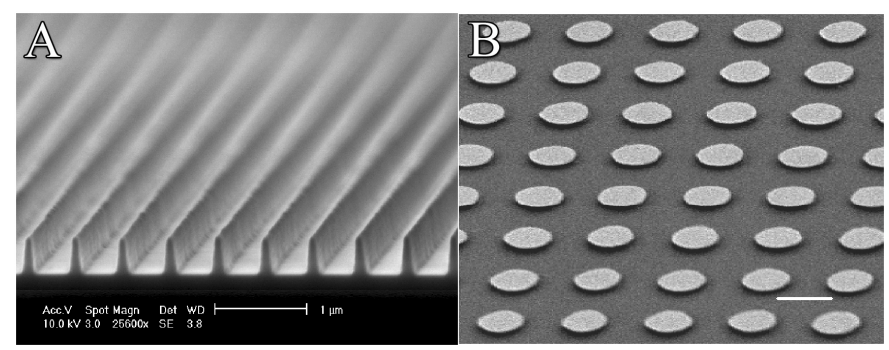

Figure 1. Laser-interference lithography was used to fabricate (a) largearea nanograting arrays on photoresist and $(b)$ gold-silver bimetallic plasmonic structures on quartz substrates. Scale bar: $1 \mu \mathrm{m}$.

a grating pattern with a period that depends on the wavelength of the light and the half angle of the intersection of the two incident beams. The line width depends on the light-exposure dose, which can be as low as 100nm: see Figure 1(a). Using this approach, after only a few minutes of UV light exposure, followed by photoresist development and chemical etching, periodic nanoline and nanodot arrays can be created in a short time on a scale of centimeters. In contrast, other single-beam direct-writing techniques require dozens of hours to fabricate equivalent large-area nanostructures.

We applied this technique to fabricate large-area plasmonic nanostructures. With the flexible tuning of LIL-exposure designs, nanodot-, nanorod-, and nanodiamond-shaped plasmonic structures can be constructed. Figure 1(b) shows such a goldsilver bimetallic structure. ${ }^{2}$ We also successfully applied this approach to create large-area 30nm-gold-dot arrays on silicon surfaces for use in growing silicon nanowires for vertical electronic devices. ${ }^{3}$ By combining LIL and catalytic etching, large-area 3D silicon nanowire and nanofin arrays can be fabricated, which can subsequently serve as molds for imprinting of electrodes for enhanced electric-field emission. ${ }^{4}$ 

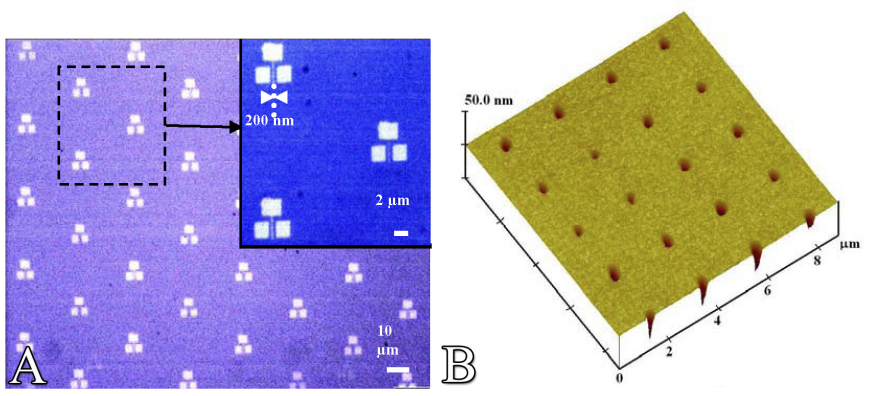

Figure 2. Laser microlens-array (MLA) lithography was used to fabricate (a) field-emission transistor arrays on a germanium antimony tellurium surface using $800 \mathrm{~nm} / 100 \mathrm{fs}$ laser irradiation through a $23 \mu \mathrm{m}$ $M L A$ and (b) nanodot arrays on photoresist using $248 \mathrm{~nm} / 23 \mathrm{~ns}$ krypton fluoride excimer-laser irradiation through a $1 \mu m \mathrm{MLA}$.

Microlens-array (MLA) lithography is another laser-based approach being developed for rapid fabrication of large-scale, ordered nanostructures. MLAs consist of a series of miniaturized lenses of identical sizes and focal lengths, typically arranged hexagonally or squarely packed. When used in a typical optical system, an MLA can focus incident light to form a series of parallel light spots in the focal plane. We used an MLA with $401 \times 401(160,801)$ lenses covering an area of $10 \times 10 \mathrm{~mm}^{2}$, with lens diameters of $23 \mu \mathrm{m}$. They were arranged hexagonally with a pitch of $25 \mu \mathrm{m}$. The sag of each lens was $9 \mu \mathrm{m}$ in height, which is equivalent to a focal length of $28.7 \mu \mathrm{m}$. Using a computercontrolled stage, we used a $800 \mathrm{~nm} / 100 \mathrm{fs}$ laser to irradiate a 30nm-thick germanium antimony tellurium layer sputtered onto a polycarbonate substrate. This generated thousands of fieldemission transistor structures in a few minutes with a gate line width of around 200nm: see Figure 2(a). In addition, using an alkaline solution, we fabricated 3D nanostructures as small as $55 \mathrm{~nm}$ on phase-change thin films. ${ }^{5}$

To achieve further reductions in feature sizes, we manufactured an MLA on a quartz substrate with a diameter and pitch of approximately $1 \mu \mathrm{m}$ each. ${ }^{6}$ The final product consisted of $2500 \times 2500$ (6.25 million) lenses covering an area of $5 \times 5 \mathrm{~mm}^{2}$. The sag of each lens was $330 \mathrm{~nm}$ in height, which is equivalent to a focal length of $1.1 \mu \mathrm{m}$. UV light-sensitive photoresist irradiated by a $248 \mathrm{~nm} / 23 \mathrm{~ns}$ krypton fluoride excimer laser through the MLA created nanodots as small as $78 \mathrm{~nm}$ with a depth of $12 \mathrm{~nm}$, at a resolution of one-third the operating wavelength: see Figure 2(b).

To extend the use of maskless multibeam-laser nanoprocessing to resolutions of less than 50nm, we are exploring using shorter-wavelength LIL, smaller MLAs, and shorter laser-pulse durations for MLA lithography. To solve the technical challenges of shallow exposure depth, we are experimenting with optimal phase-change materials as hard masks for transferring nanopatterns onto the substrates. Together, these advances should promote using maskless multibeam laser irradiation for rapid fabrication of large-area nanodevices.

\section{Author Information}

Minghui Hong, Leng Seow Tan, Boris Luk'yanchuk, Luping Shi and Tow Chong Chong

Department of Electrical and Computer Engineering

National University of Singapore (NUS)

Singapore, Singapore

and

Data Storage Institute

Singapore, Singapore

Minghui Hong is an associate professor at NUS and a senior scientist at the Data Storage Institute, A*STAR.

Leng Tan is an associate professor at NUS.

Boris Luk'yanchuk is a senior scientist at A*STAR.

Luping Shi is a principal scientist and division manager at A*STAR.

Tow Chong is a full professor at NUS and the executive director of $A *$ STAR.

References

1. T. C. Chong, M. H. Hong, and L. P. Shi, Laser precision engineering: from microfabrication to nanoprocessing, Laser Photon. Rev. in press, doi:10.1002/lpor.200810057

2. C. H. Liu, M. H. Hong, H. W. Cheung, F. Zhang, Z. Q. Huang, L. S. Tan, and T. S. A. Hor, Bimetallic structure fabricated by laser interference lithography for tuning surface plasmon resonance, Opt. Express 16, pp. 10701-10709, 2008.

3. W. K. Choi, T. H. Liew, M. K. Dawood, I. S. Henry, C. V. Thompson, and M. H. Hong, Synthesis of silicon nanowires and nanofin arrays using interference lithography and catalytic etching, Nano Lett. 8, pp. 3799-3802, 2008.

4. W. K. Choi , T. H. Liew, H. G. Chew, F. Zheng, C. V. Thompson, Y. Wang, M. H. Hong, X. D. Wang, L. Li, and J. Yun, A combined top-down and bottom-up approach for precise placement of metal nanoparticles on silicon, Small 4, pp. 330-333, 2008 5. Y. Lin, M. H. Hong, T. C. Chong, C. S. Lim, G. X. Chen, L. S. Tan, Z. B. Wang, and L. P. Shi, Ultrafast-laser-induced parallel phase-change nanolithography, Appl. Phys. Lett. 89, p. 041108, 2006

6. C. S. Lim, M. H. Hong, Y. Lin, Q. Xie, B. S. Luk'yanchuk, A. Senthil Kumar, and M. Rahman, Microlens array fabrication by laser interference lithography for superresolution surface nanopatterning, Appl. Phys. Lett. 89, p. 191125, 2006. 